\title{
Inhalation von Tobramycin über 12 Monate zur Senkung der Hospitalisations- rate bei schwerer COPD mit gehäuften Hospitalisationen
}

\author{
Effect of Inhalation of Tobramycin for 12 Months on Reduction of Hospitalisation Rate in Severe COPD
}

Autoren

Institute
P. Haidl ${ }^{1}$, J. Bargon ${ }^{2}$, T. Gessler ${ }^{3}$, M. Pfeifer ${ }^{4}$, W. Randerath ${ }^{5}$, T. Voshaar ${ }^{6}$, B. Wachall

Die Institutsangaben sind am Ende des Beitrags gelistet. eingereicht 13.5 .2013 akzeptiert nach Revision 4.6.2013

Bibliografie

Dol http://dx.doi.org/ 10.1055/s-0033-1344344 Online-Publikation: 8.7.2013 Pneumologie 2013; 67: 514-519 (c) Georg Thieme Verlag KG Stuttgart · New York ISSN 0934-8387

Korrespondenzadresse Dr. Peter Haidl

Fachkrankenhaus Kloster Grafschaft

Abteilung Pneumologie II Annostr. 1 57392 Schmallenberg p.haidl@fkkg.de

\section{Zusammenfassung \\ V}

Fragestellung: Eine bakterielle Kolonisation im stabilen Intervall der COPD und Bronchiektasen können Ursachen für wiederholte, prognostisch ungünstige Hospitalisationen sein. Die Studie untersuchte den Einfluss der Inhalation von Tobramycin auf die Häufigkeit von Hospitalisationen bei Patienten mit COPD und mindestens 2 Hospitalisationen im Jahr vor Studieneinschluss.

Methode: 44 Patienten mit COPD $\left(\mathrm{FEV}_{1} \%\right.$ Sollwert: 42,8 \pm 7,1 Tobramycin (T); 33,5 $\pm 10,3$ Placebo $(\mathrm{P})$ ) inhalierten (randomisiert, doppelblind) über ein Jahr täglich zweimal $80 \mathrm{mg}$ Tobramycin oder Placebo „add on“ zur Guideline-Therapie. Primärer Endpunkt: Zahl der COPD-Hospitalisationen. Sekundäre Endpunkte: Lungenfunktion und 6-Minuten-Gehstrecke.

Ergebnisse: In der T-Gruppe betrug die Zahl der COPD-Hospitalisationen 2,8 $\pm 0,5 \mathrm{im}$ Jahr vor Studieneinschluss und $3,5 \pm 2,7$ im Studienjahr, in der P-Gruppe 3,0 $\pm 1,4$ und 2,3 $\pm 2,2$. Der Unterschied war nicht signifikant, ebenso nicht für die sekundären Endpunkte. Hohe Abbruchrate mit 24 von 44 Patienten. Nur 6 Patienten in der Tund 14 Patienten in der P-Gruppe beendeten die Studie protokollgerecht.

Schlussfolgerung: Die 12-monatige tägliche Inhalation von $160 \mathrm{mg}$ Tobramycin mit einem Vernebler führte nicht zu einer Reduktion der Zahl der Hospitalisationen im Vergleich zu Placebo. Die hohe Abbruchrate war überwiegend bedingt durch die Schwere der Grunderkrankung.

\section{Einleitung}

$\nabla$

Die Senkung der Exazerbationsrate ist bei der COPD ein klinisch bedeutsames Therapieziel, das die Prognose der Erkrankung maßgeblich beeinflusst. [1]

\section{Abstract \\ $\nabla$}

Introduction: Bacterial colonisation in stable disease of severe COPD and bronchiectasis can cause recurrent hospital treatment, which has a negative impact on the patient's prognosis. A multicentre study has investigated if daily inhalation of tobramycin for one year would lower the number of hospitalisations in severe COPD.

Methods: 44 patients with severe COPD $\left[\mathrm{FEV}_{1} \%\right.$ of predicted value: $42.8 \pm 7,1$ tobramycin group $(\mathrm{T})$ and $33.5 \pm 10.3$ placebo group $(\mathrm{P})]$ and a minimum of two hospitalisations in the year before inclusion were randomly assigned to inhale twice daily for 12 months $80 \mathrm{mg}$ tobramycin or isotonic saline (placebo). Concomitant therapy was according to the GOLD guidelines. Primary end point was the number of hospitalisations in the period of study, secondary end points were pulmonary function test and 6 MWD.

Results: Inhalation of $\mathrm{T}$ changed the number of hospitalisations from $2.8 \pm 0.5$ per year to $3.5 \pm$ 2.7, P from $3.0 \pm 1,4$ to $2.3 \pm 2.2$. These differences and the results for secondary endpoints did not reach significance. The dropout rate was high, only 6 patients $(T)$ and 14 patients $(P)$ finished the study per protocol.

Conclusion: Inhalation with $160 \mathrm{mg}$ tobramycin by means of a nebuliser over a 12-month period did not reduce the number of hospitalisations for patients with severe COPD and a minimum of two hospitalisations compared to placebo. The severity of the disease was the main reason for the high dropout rate.

Verschiedene medikamentöse Interventionen mit inhalativen Anticholinergika und fixen Kombinationen aus langwirksamen Beta-Sympathomimetika und Steroiden sowie die orale Gabe von Ro-

\footnotetext{
* Der Beitrag ist Prof. Dr. med. Dieter Köhler zum 65.Geburtstag gewidmet.
} 
flumilast konnten Reduktionen der jährlichen Exazerbationen um ca. $20 \%$ erzielen [2 -5]. Dabei führt nur ca. jede 8 . Exazerbation zu einer Krankenhauseinweisung. Wenn es im Rahmen einer COPD zu einer Hospitalisation aufgrund einer Infektexazerbation kommt, ist dies mit einer deutlichen Verschlechterung der Prognose verbunden. Soler-Cataluna et al. [6] zeigten, dass nach 5 Jahren nur noch 20\% der COPD-Patienten lebten, wenn diese mehr als 2 stationäre Aufnahmen pro Jahr erfahren hatten.

Die erhöhte Rate an Exazerbationen scheint verschiedene Ursachen zu haben, unter anderem die Schwere der neutrophilen Entzündung, die Besiedelung mit gramnegativen Keimen, das Auftreten von Bronchiektasen und die Verschlechterung der bronchialen Reinigung durch die zunehmende Immobilität [7, 8]. Insbesondere die bakterielle Kolonisation der unteren Atemwege spielt eine bedeutsame Rolle und geht mit einer erhöhten Entzündungsaktivität einher [9]. Die Rate von Exazerbationen wird erhöht, der jährliche $\mathrm{FEV}_{1}$-Abfall beschleunigt [10]. Möglicherweise führen virale und bakterielle Infekte zu einer Epithelzerstörung, die dann im Verlauf durch die Reduktion protektiver Faktoren (reduzierte mukoziliäre Clearance, verminderte Sekretion, Neutralisierung der Immunglobuline, reduzierte Phagozytenaktivität) den Übergang der Kolonisation in eine akute Exazerbation zur Folge haben können [11].

Daher stellten wir die Hypothese auf, dass durch die regelmäßige Inhalation eines Aminoglykosids dieser Prozess gehemmt bzw. verzögert wird. Die Inhalation von Tobramycin erreicht eine 25bis 100-fach höhere Medikamentenkonzentration im Sputum im Vergleich zur intravenösen Gabe bei kaum messbaren Serumspiegeln [12]. Unterstellt wurde, dass die erhöhte Hospitalisationsrate hauptsächlich durch eine bakterielle Kolonisationsrate im stabilen Intervall bedingt war, auch wenn zum Studieneinschluss nach in der Regel durchgeführter Pseudomonas-wirksamer Antibiose kein Keimnachweis vorlag. Untersucht wurden dabei schwerkranke COPD-Patienten mit schlechter Prognose und hohem Ressourcenverbrauch, gekennzeichnet durch eine Hospitalisationsrate von mindestens 2 pro Jahr. Diese Patientengruppe scheidet häufig aus den o.g. Studien aus, sodass hier keine validen Daten vorliegen.

Verglichen wurde, ob die dauerhafte Inhalation von Tobramycin über 12 Monate im Unterschied zu Placebo als „,add on“ zur Standardtherapie zu einer Veränderung der Zahl exazerbationsbedingter Krankenhauseinweisungen führt.

\section{Methode}

\section{$\nabla$}

Im Parallelgruppendesign wurde eine prospektive, multizentrische, doppelblinde, placebokontrollierte „add on“-Phase-III-Studie durchgeführt.

Die Teilnehmer wurden in einem 1:1-Verhältnis randomisiert, entweder der Verum-Gruppe (Inhalation von Tobramycin $2 \times$ täglich 1 Ampulle Gernebcin ${ }^{\circledR} 80 \mathrm{mg}$ (Firma Infectopharm, Heppenheim) verdünnt in $2,5 \mathrm{ml}$ physiologischer $\mathrm{NaCl}$-Lösung) oder der Placebo-Gruppe (2× täglich 4,5 ml physiologische NaCl-Lösung) zugeordnet.

Die Randomisierungsliste wurde mit der aktuellen Version der Randomisierungssoftware RANDOM der Ecron Acunova GmbH erstellt

Die Inhalation erfolgte mit dem Verneblersystem Pariboy SX/Pari LC Sprint mit Kontrolle des Inspirationsflusses und, soweit toleriert, vorgegebenem Widerstand in der Ausatemphase (PIF-Con- trol und PEP-System, Fa. Pari GmbH, München). Die geplante Behandlungsdauer betrug 12 Monate.

Eine Fallzahl von 80 Patienten wurde kalkuliert. Unterstellt wurde aufgrund einer retrospektiven Patientenanalyse aus dem Fachkrankenhaus Kloster Grafschaft, dass die Zahl der Hospitalisationen um 30\% in einem Jahr gegenüber Placebo gesenkt werden könnte. Weiterhin wurde eine Standardabweichung von 1 angenommen. Sechs Zentren nahmen an der Rekrutierung der Patienten teil, von allen Studienpatienten lag eine schriftliche Einverständniserklärung vor.

Die Studie wurde von der Ethikkommission der Medizinischen Fakultät der Westfälischen Wilhelms-Universität Münster genehmigt.

\section{Studienteilnehmer}

Im Zeitraum von Februar 2009 bis September 2010 wurden 44 Patienten mit schwerer bis sehr schwerer COPD (Stadium III bis IV nach GOLD) mit einem $\mathrm{FEV}_{1} \leq 50 \%$ Sollwert und einem $\mathrm{FEV}_{1} /$ FVC $<70 \%$ eingeschlossen. Das Studienende des letzten Patienten war Juni 2011. Die Patienten waren zwischen 40 und 79 Jahre alt und seit mindestens 6 Monaten Nichtraucher. Die Dauertherapie der COPD musste in den letzten 4 Wochen vor Einschluss mindestens 2 der folgenden 3 Therapeutika enthalten: Tiotropiumbromid, inhalatives Steroid, langwirksames Beta-Sympathomimetikum. Die Patienten waren in den letzten 12 Monaten vor Studienbeginn mindestens 2-mal wegen ihrer Grunderkrankung hospitalisiert (die Hospitalisation zum Zeitpunkt des Studieneinschlusses wurde dabei mit eingerechnet).

\section{Ausschlusskriterien}

Wesentliche Ausschlusskriterien waren eine Antibiotika-Inhalation innerhalb der letzten 6 Monate oder die Inhalation von Gernebcin in der Vergangenheit. Weiterhin führten eine Sauerstofflangzeittherapie oder eine nicht invasive Beatmung, die in den letzten 3 Monaten vor Studienbeginn begonnen wurde, zum Ausschluss, da dies die Exazerbationsrate beeinflusst hätte. Eine systemische Kortikosteroid-Dauertherapie über $20 \mathrm{mg}$ Prednisolonäquivalent führte ebenso zum Ausschluss wie der Nachweis einer Niereninsuffizienz mit einem Kreatininwert über $2 \mathrm{mg} / \mathrm{dl}$.

\section{Ablauf des Studieneinschlusses}

Während des stationären Aufenthaltes, spätestens jedoch 3 Tage vor der geplanten Entlassung, erfolgte der Einschluss der Patienten nach Prüfung der Ein- und Ausschlusskriterien. Während der stationären Routinebehandlung wurde die übliche, begleitende Inhalation von Kochsalzlösung mit einem „Übungs-Pariboy“ durchgeführt. Damit wurde sichergestellt, dass die Inhalation mit einem Vernebler toleriert wurde. Gleichzeitig wurde die für die Inhalation benötigte Zeit dokumentiert, da diese als Grundlage für die Compliance-Kontrolle benötigt wurde. Am Morgen des letzten Tages vor der Entlassung wurde neben der Erfassung der Lungenfunktionsparameter, der Laborwerte und der 6-MinutenGehstrecke eine Inhalation der patientenspezifischen Prüfmedikation durchgeführt. Diese erfolgte frühestens zwei Stunden nach der morgendlichen Inhalation eines kurz- oder langwirksamen Betamimetikums. Im unmittelbaren Anschluss an die Inhalation mit der Prüfmedikation wurde eine Spirometrie durchgeführt. Falls es zu einem Abfall von mehr als 20\% des Ausgangs$\mathrm{FEV}_{1}$ kam, wurde der Patient aus der Studie ausgeschlossen. Eine Stunde nach der Probeinhalation der Prüfmedikation erfolgte eine weitere Blutentnahme zur Bestimmung des TobramycinSerumspiegels. 
Falls der Patient am Entlassungstag Sputum generieren konnte, wurde dies in das jeweilige lokale Labor gesendet und bei positivem bakteriologischen Befund zur Resistenzbestimmung an das Referenzlabor Dr. M. Kresken (Antiinfectives Intelligence $\mathrm{GmbH}$, 53359 Rheinbach) weitergeleitet.

Der Prüfarzt erhielt nach Bestimmung des Tobramycinspiegels einen schriftlichen, anonymisierten Bericht, der auswies, ob der Tobramycinspiegel über oder unter dem Grenzwert von $2 \mu \mathrm{g} / \mathrm{ml}$ (Talspiegel der iv-Therapie) lag. Für den Fall, dass der erlaubte Grenzwert überschritten wurde, erfolgte der Studienausschluss des Patienten.

Nach 4 Wochen wurde der Kompressor des Verneblers per Post ausgetauscht und die Betriebsstundenzahl des eingebauten Zählers ausgelesen. Bei einem Wert von weniger als 50\% des individuell aus den Probeinhalationszeiten berechneten Sollwertes wurde der Patient von der weiteren Teilnahme ausgeschlossen. 3, 6 und 9 Monate nach Einschluss setzte sich der Prüfarzt mit dem Patienten telefonisch in Verbindung. Erfragt wurden anhand eines Interviewbogens subjektives Befinden, evtl. Exazerbationen, Hospitalisationen, ungeplante Arztbesuche und unerwünschte Ereignisse. Schließlich füllten die Teilnehmer täglich einen Fragebogen zu Symptomen, ungeplanten Arztbesuchen und einer Änderung der Medikation aus. Diese wurden monatlich dem Prüfarzt zugesandt.

Bei Studieneinschluss und bei jeder stationären Aufnahme wegen einer Exazerbation der COPD wurde versucht, Sputum zu gewinnen und eine mikrobiologische Untersuchung zu veranlassen. Falls die stationäre Aufnahme nicht im Prüfzentrum erfolgte, war der Patient angehalten, eine Kontaktaufnahme der betreuenden Klinik mit dem Prüfzentrum herzustellen.

Nach 12 Monaten erfolgte eine ambulante Abschlussuntersuchung (im Intervall, d.h. außerhalb einer Exazerbation). Erfasst wurde die Zahl der im Prüfzeitraum erfolgten Hospitalisationen, darüber hinaus wurden Laboruntersuchungen, Lungenfunktion und die 6-Minuten-Gehstrecke erfasst.

\section{Zielkriterien}

Primäre Zielgröße war die Häufigkeit der Hospitalisationen im Jahr der Studie infolge einer Exazerbation der COPD (normiert auf ein Jahr at risk).

Sekundäre Zielparameter waren Lungenfunktionsparameter (insbesondere $\mathrm{FEV}_{1}, \mathrm{RV} / \mathrm{TLC}$ in \% und intrathorakales Gasvolumen) sowie die 6-Minuten-Gehstrecke. Weiter ausgewertet wurden die Keimbestimmung im Sputum zu Beginn und im Verlauf der Studie.

\section{Statistik}

Für die Auswertung wurde die SAS Software (statistical analysis system von SAS Institute Incorporation (CARY NC)) in der Version 9.1 verwendet. Auswertepopulation waren alle Patienten, die mindestens einmal Prüfmedikation erhielten (inklusive der Probeinhalation der Prüfmedikation (safety-evaluable-populationSE-Population). Die Intention-to-treat-Population ITT 0 beinhaltete alle randomisierten Patienten, die die Probeinhalation der Prüfmedikation tolerierten und für die Daten zum primären Zielparameter vorhanden waren. Die Intention-to-treat-Population ITT 1 beinhaltete alle Patienten der ITT-0-Population, die mindestens 28 Tage Prüfmedikation inhaliert hatten. Zur per-Protokollauswertung (PP-Population) gehörten alle Patienten der ITT1-Population, die keine relevanten Protokollverletzungen aufwiesen (z.B. Beobachtungsdauer von weniger als 11 Monaten, orale oder intravenöse Antibiotikatherapie über mehr als ein Drittel der Beobachtungsdauer, schlechte Compliance).

Verwendet wurde für verbundene Stichproben der WilcoxonRangsummen-Test. Alle statistischen Tests wurden zweiseitig durchgeführt mit einem Signifikanzniveau $\alpha=0.05$.

\section{Ergebnisse \\ $\nabla$}

44 Patienten wurden in die Studie eingeschlossen. 41 Patienten inhalierten mindestens eine Dosis der Prüfmedikation ( $\bullet$ Tab. 1). Neun Patienten schieden bis zum Zeitpunkt der Klinikentlassung aus. Tab. 2 zeigt die Ursachen für diese hohe „drop-out“-Rate. Die biometrischen Daten und die Lungenfunktionsparameter der verbleibenden 32 Patienten (ITT 0) zeigt $\bullet$ Tab.3. Zur Auswertung in der ITT-1-Population, die für die Primäranalyse herangezogen wurde, gelangten 30 Patienten, in die per-Protokoll-Gruppe (PP) 20 Patienten ( Tab.1). In $\bullet$ Tab. 4 sind die Gründe für weitere Abbrüche im Zeitraum von 4 Wochen nach Studienbeginn (ITT 1) bis Studienende (PP) aufgeführt.

Tab. 1 Fallzahlen der unterschiedlichen Auswerte-Populationen.

\begin{tabular}{|lll|} 
& Verum & Placebo \\
\hline Safety $(n)$ & 18 & 23 \\
\hline ITT 0 $(n)$ & 11 & 21 \\
\hline ITT 1 $(n)$ & 9 & 21 \\
\hline PP $(n)$ & 6 & 14 \\
\hline
\end{tabular}

Tab.2 Abbruchgründe zwischen Studieneinschluss (Safety) und Entlassung aus der stationären Behandlung (ITT 0).

\begin{tabular}{|lll|}
\hline & Verum & Placebo \\
\hline Verletzung Einschlusskriterien & 1 & 0 \\
\hline unerwünschte Ereignisse (Luftnot) & 2 & 2 \\
\hline Rücknahme des Einverständnisses & 2 & 0 \\
\hline Obstruktion nach Probeinhalation & 2 & 0 \\
\hline
\end{tabular}

Tab. 3 Biometrische und Lungenfunktionsparameter (ITT 0).

\begin{tabular}{|lll|}
\hline & Verum & Placebo \\
\hline N & 11 & 21 \\
\hline Alter $(\mathrm{J})$ & $65,5 \pm 4,7$ & $63,9 \pm 9,4$ \\
\hline BMI $\left(\mathrm{kg} / \mathrm{m}^{2}\right)$ & $23,7 \pm 4,8$ & $24,0 \pm 5,0$ \\
\hline Geschlecht $(\% w)^{\text {FEV }}$ (\%Soll) & 45 & 33 \\
\hline ITGV $(\%$ Soll) & $41,6 \pm 7.0$ & $33,5 \pm 10.3$ \\
\hline RV/TLC $(\%$ Soll) & $148 \pm 28.6$ & $174,4 \pm 55,6$ \\
\hline 6 MWD $(\mathrm{m})$ & $128,4 \pm 42,7$ & $166,4 \pm 43,0$ \\
\hline
\end{tabular}

$\mathrm{FEV}_{1}$ : forciertes expiratorisches Volumen in einer Sekunde; ITGV: intrathorakales Gasvolumen; RV/TLC: Residualvolumen in Prozent der totalen Lungenkapazität.

Tab. 4 Patienten mit Protokollverletzungen nach mindestens 4-wöchiger Studienteilnahme bis zum Studienende.

\begin{tabular}{|lll|}
\hline & Verum & Placebo \\
\hline Beobachtungsdauer unter 180 Tagen & 2 & 4 \\
\hline Compliance unter 80 \% der Prüfmedikation & 1 & 3 \\
\hline Summe & 3 & 7 \\
\hline
\end{tabular}


Tab.5 Zahl der Hospitalisationen vor und während der Studie.

\begin{tabular}{|lll} 
& Verum & Placebo \\
\hline $\begin{array}{l}\text { Hospitalisation im Jahr vor } \\
\text { Studieneinschluss }(\mathrm{n})\end{array}$ & $2,8 \pm 0,5$ & $3,0 \pm 1,4$ \\
\hline ITT 1 $(\mathrm{n})$ & $4,3 \pm 2,6$ & $2,5 \pm 1,9$ \\
\hline PP $(\mathrm{n})$ & $3,5 \pm 2,7$ & $2,3 \pm 2,2$ \\
\hline
\end{tabular}

Tab. 6 Häufigkeitsverteilung der Hospitalisationen in den letzten 12 Monaten vor Studienbeginn (ITT1 Population).

\begin{tabular}{|lccll|}
$\begin{array}{l}\text { Hospitalisationen im Jahr } \\
\text { vor Studieneinschluss (n) }\end{array}$ & $\begin{array}{l}\text { Placebo } \\
\text { (n) }\end{array}$ & $\begin{array}{l}\text { (\%) } \\
\text { Verum }\end{array}$ & $\begin{array}{l}\text { (\%) } \\
\text { (n) }\end{array}$ \\
\hline 2 & 11 & 52 & 3 & 33 \\
\hline 4 & 5 & 24 & 5 & 56 \\
\hline 5 & 3 & 14 & 1 & 11 \\
\hline 9 & 1 & 5 & 0 & 0 \\
\hline
\end{tabular}

Tab. 7 Ergebnisse der sekundären Zielparameter.

\begin{tabular}{|lllll|}
\hline & Verum & Placebo & Verum & Placebo \\
\hline & ITT 1 & ITT 1 & PP & PP \\
\hline FEV $_{1}$ (\%Soll) & $30,9 \pm 9,4$ & $31,8 \pm 8,7$ & $30,4 \pm 10,1$ & $32,5 \pm 8,8$ \\
\hline ITGV (\%Soll) & $165,6 \pm 29,1$ & $182,2 \pm 34,0$ & $182,2 \pm 34,0$ & $174,4 \pm 55,6$ \\
\hline RV/TLC (\%Soll) & $176,3 \pm 22,8$ & $170,7 \pm 39,0$ & $179,2 \pm 23,5$ & $166,0 \pm 37,2$ \\
\hline 6 MWD (m) & $272 \pm 86$ & $301 \pm 181$ & $259 \pm 86$ & $281 \pm 174$ \\
\hline
\end{tabular}

ITGV = intrathorakales Gasvolumen, RV/TLC=Verhältnis des Residualvolumens zur totalen Lungenkapazität, 6 MWD = 6-Minuten-Gehstrecke.

Die Zahl der Hospitalisationen im Jahr vor Studieneinschluss war in beiden Gruppen vergleichbar (Verum 2,8 $\pm 0,5$; Placebo $3,0 \pm 1,4$ ). Sowohl in der ITT-1- als auch der PP-Population kam es nicht zu einer signifikanten Veränderung der Hospitalisationsrate ( Tab.5). Allerdings war die Verteilung der Hospitalisationen vor Studieneinschluss in den beiden Gruppen etwas heterogen (in der Placebogruppe finden sich sowohl mehr leichtere Fälle als auch die 2 Patienten mit den höchsten Hospitalisationsraten, 5 und 9), was sich auch in der tendenziell höheren Hospitalisationsrate der Verumgruppe in der PP-Population widerspiegelt ( $\bullet$ Tab.6).

Die sekundären Endpunkte ( $\mathrm{FEV}_{1}$, RV/TLC, ITGV, 6 MWD) zeigten ebenfalls keine signifikanten Abweichungen zwischen den Gruppen ( $\bullet$ Tab.7). Die Patienten in der Placebo-Gruppe wiesen jedoch tendenziell ein etwas höheres Ausmaß an Obstruktion und eine stärkere Überblähung in den Lungenfunktionsparametern (z.B. RV/TLC) auf, was für eine im Mittel etwas schwerere Erkrankung der Placebo-Patienten spricht.

Während der Studie verstarben 5 Patienten, davon 2 in der Verum- und 3 in der Placebo-Gruppe. 2 Patienten verstarben in der Woche nach Studienende, jeweils ein Patient in jeder Gruppe. Bei keinem der Todesfälle wurde ein Kausalzusammenhang mit der Studienmedikation dokumentiert.

Der Anteil der Patienten mit Bronchiektasen war gering (Verum $27 \%$, Placebo $10 \%$ ).

Kein Patient in der Verum-Gruppe wies nach der Probeinhalation einen erhöhten Tobramycin-Serumspiegel auf.
Keimnachweis:

In der ITT-1-Population konnte bei 7 der 9 Verum-Patienten ein Sputum gewonnen werden. In 4 Fällen konnten Keime nachgewiesen werden, davon einmal Pseudomonas aeruginosa. In der Placebo-Gruppe gelang der Keimnachweis bei 11 von 13 Patienten (gesamt 21 Pat.), davon zweimal Pseudomonas aeruginosa. Der häufigste Keim in beiden Gruppen war Hämophilus influenzae (5 Fälle).

In der Placebo-Gruppe fanden sich im Verlauf Resistenzen gegen Tobramycin für Streptokokkus pneumoniae (1) und Streptokokkus oralis (2). Bei jedem der 4 Patienten der Verum-Gruppe fanden sich opportunistische Keime (3-mal Streptokokkus oralis, einmal Enterokokkus faecalis), die jeweils gegen Tobramycin resistent waren (MHK $32 \mathrm{mg} / \mathrm{l})$.

Verträglichkeit:

Unerwünschte Ereignisse traten bei 30 von 41 Patienten auf (Erkrankungen der Atemwege, des Gastrointestinaltraktes und Herzerkrankungen). Diese waren in beiden Behandlungsgruppen gleich häufig. Insgesamt wurde die Studienmedikation bei $6 \mathrm{~Pa}-$ tienten in der Tobramycingruppe und bei 4 Patienten in der Placebogruppe aufgrund von unerwünschten Ereignissen abgesetzt. Schwere unerwünschte Ereignisse wurden in 23 Fällen dokumentiert (Tobramycin 27,8\%, Placebo 39,1\%). Ein Zusammenhang mit der Studienmedikation wurde in keinem Fall hergestellt.

\section{Diskussion}

Die vorgelegte randomisierte und doppelblinde Studie zeigte, dass durch die regelmäßige Inhalation von Tobramycin über 12 Monate im Vergleich mit Placebo bei Patienten mit schwerer COPD keine Senkung der exazerbationsbedingten Krankenhauseinweisungen erzielt wurde. Die sekundären Zielparameter Lungenfunktion und 6-Minuten-Gehstrecke ergaben ebenfalls keine Überlegenheit der Tobramycin-Gruppe. Es handelt sich hier um die erste Studie in der Literatur, die sich dieser Fragestellung widmet.

Die Aussagekraft der Studie ist eingeschränkt, da zum einen die geplante Patientenzahl von 80 nicht erreicht wurde, zum anderen die Zahl der vorzeitigen Studienabbrüche mit 24 von $44 \mathrm{~Pa}-$ tienten sehr hoch war. Hauptgrund war nur in 2 Fällen eine durch Tobramycin ausgelöste Bronchialobstruktion. In je 2 Fällen in der Tobramycin- und in der Placebogruppe führte die Vernebler-Inhalation zu Luftnot. In der Mehrzahl der Fälle waren die Abbrüche durch Tod oder die Schwere der Erkrankung bedingt, die die Patienten in der Einhaltung des Studienprotokolls behinderte ( $\odot$ Tab.2 und $\odot$ Tab.4). Auch die Rekrutierung der Studienteilnehmer wurde durch die Schwere der Grunderkrankung maßgeblich beeinflusst.

Die dauerhafte Gabe von Antibiotika ist Gegenstand aktueller Diskussionen in der Therapie der COPD. Für die Inhalation, insbesondere von Tobramycin, liegen umfangreiche positive Erfahrungen in der Therapie der zystischen Fibrose $[13,14]$ vor.

Studien zur Inhalation von Antibiotika bei COPD existieren hingegen bisher nicht [15]. Dagegen sind inzwischen verschiedene Untersuchungen zur Antibiotika-Inhalation mit Tobramycin und Ciprofloxacin bei non-CF Bronchiektasen [16-19] publiziert worden.

Murray et al. [20] führten in einem solchen Kollektiv von $65 \mathrm{~Pa}-$ tienten eine 12-monatige Studie durch, in der kontinuierlich $160 \mathrm{mg}$ Gentamicin per Vernebler gegen Placebo geprüft wurde. 
Zielparameter waren die Reduktion der Keimzahl im Sputum sowie die Sputummenge, sekundäre Kriterien waren u.a. die Exazerbationsrate und Lungenfunktionsparameter. Die GentamicinInhalation konnte die Keimzahl im Verlauf signifikant reduzieren, die Eradikation von Pseudomonas gelang in 31\% der Fälle, und die Exazerbationsrate sank signifikant. Resistenzen wurden nicht beobachtet. Die Wirksamkeit ließ in der dreimonatigen therapiefreien Nachbeobachtungsperiode nach.

Die Abbruchrate war bei vergleichbarem Studienprotokoll deutlich geringer als in unserer Studie, je drei in jeder Gruppe sowie zwei Todesfälle. Möglicherweise sind die Patienten mit non-CF Bronchiektasen gesünder und weisen eine geringere Komorbidität auf. Der $\mathrm{FEV}_{1}$ \% Sollwert betrug in der zitierten Studie 73\% (Gentamicin) und 63\% (Placebo).

Einschlusskriterium in der Murray-Studie war der mindestens dreimalige Keimnachweis im stabilen Intervall in den letzten 12 Monaten.

In unserer Untersuchung war dies nicht gefordert. Dies war auch der Praktikabilität und den Kosten geschuldet. Wenn der Keimnachweis im stabilen Intervall geführt worden wäre, hätten sich die Patienten nochmals im Prüfzentrum vorstellen müssen, und der Studieneinschluss wäre unter ambulanten Bedingungen erfolgt. Aufgrund der Schwere der Grunderkrankung hätten mehr Patienten wegen des Aufwandes die Studienteilnahme abgelehnt. Daher wählten wir die Hospitalisationsrate als Hauptkriterium und führten den Studieneinschluss mit Ende eines Krankenhausaufenthaltes durch.

Albert et al. [21] untersuchten den Einfluss der dauerhaften Einnahme von $250 \mathrm{mg}$ Azithromycin auf die Senkung der Exazerbationsrate bei 1142 Patienten mit COPD und schwerer Obstruktion $\left(\mathrm{FEV}_{1}\right.$ \%Soll 39 bzw. 40). Eingeschlossen wurden auch Patienten mit einer Sauerstoff-Langzeittherapie, weitere Kriterien waren eine Notfallbehandlung oder eine Hospitalisation im Vorjahr. Die Zahl der Probanden, bei denen eine Sputumanalyse erfolgen konnte, war zu gering, sodass dafür Nasopharynx-Abstriche durchgeführt wurden. Die Adhärenz mit der Studienmedikation betrug ca. $67 \%$ in beiden Gruppen. Die Todesfallrate betrug 3\% bzw. 4\% in beiden Gruppen, die Zahl der Abbrüche 32 (Azithromycin) und 28 (Placebo). Die Dauergabe von Azithromycin senkte die Exazerbationsrate im Vergleich zu Placebo um 27\%, die absolute Reduktion betrug 37\% in der Azithromycin-Gruppe und $16 \%$ in der Placebo-Gruppe. Die Zahl der Krankenhauseinweisungen wurde im Vergleich zu Placebo nicht gesenkt (0,34 zu 0,49 Einweisungen pro Patientenjahr). Die Zahl der Kulturen mit einer Resistenz gegenüber Makroliden betrug 52\% (Azithromycin) und 57\% (Placebo) und stieg im Studienverlauf. Als Nebenwirkung wurde eine Abnahme des Hörvermögens beobachtet.

Die Ergebnisse legen den Schluss nahe, dass der Effekt der Makrolide weniger antibakteriell als antiinflammatorisch sein dürfte. Eine weitere Studie zu COPD und Dauergabe von Erythromycin bei 109 Patienten von Seemungal et al. senkte die Exazerbationsrate um 35\% im Vergleich zu Placebo. Hier wurde im Gegensatz zur Arbeit von Murray diskutiert, dass der antimikrobielle Effekt überwiegen würde bei fehlender Änderung der inflammatorischen Marker im Verlauf eines Jahres [22].

Limitationen:

Die Studienhypothese, dass eine bakterielle Kolonisation die Zahl der Krankenhauseinweisungen durch Exazerbationen erhöht, konnte nicht bestätigt werden. Eine strengere Fassung der Einschlusskriterien wäre sinnvoll gewesen. In der vorliegenden Studie war der Nachweis der Kolonisation im stabilen Intervall nicht gefordert, wie dies bei der Untersuchung von Murray der
Fall war [20]. Dies hätte eine weitere Reduktion der Patientenzahlen bei ohnehin schwieriger Rekrutierung zur Folge gehabt. Dazu wäre der Einschluss in den ambulanten Bereich verschoben worden mit dann erschwerter Logistik. Weiter wäre zu diskutieren, nur Patienten mit wenig ausgeprägten Bronchiektasen („Minor-Bronchiektasen“) aufzunehmen. So konnte Patel et al. [23] einen Zusammenhang zwischen mäßig ausgeprägten Bronchiektasen der Unterlappen, bakterieller Kolonisation und der Zahl schwerer Exazerbationen zeigen. In der vorliegenden Untersuchung mit einer retrospektiven Analyse der CT-Befunde lag der Anteil der Patienten mit Bronchiektasen bei 27\% (Verum) bzw. 10\% (Placebo).

Zusammenfassend führte die Inhalation von Tobramycin bei schwerer COPD nicht zur Senkung der Anzahl an Krankenhauseinweisungen. Der fehlende Effekt ist mitbedingt durch die hohe Ausfallrate der Patienten insbesondere kurz nach Studienbeginn. Hauptgrund war die Schwere der Grunderkrankung, die die Studienadhärenz beeinflusste und nicht die Nebenwirkungen der Tobramycin-Inhalation. Der Nachweis einer bakteriellen Kolonisation im stabilen Intervall der COPD und von Bronchiektasen sollten bei zukünftigen Studienplanungen zu den Einschlusskriterien gehören. Nach Einschätzung der Autoren sollte die Häufigkeit der Inhalation auf einmal täglich reduziert werden unter Verwendung möglichst effizienter Inhalationssysteme. Ob eine „on-off"-Verabreichung im monatlichen Wechsel wie bei der zystischen Fibrose vorteilhaft wäre, kann hier nicht beantwortet werden.

Die Problematik der Senkung der Exazerbationsrate bei dieser kleinen, aber schwer kranken Patientengruppe mit schlechter Prognose sollte dennoch Gegenstand weiterer Forschung sein.

\section{Interessenkonflikt}

$\nabla$

B. Wachall ist Mitarbeiter der Firma Infectopharm Arzneimittel $\mathrm{GmbH}$. Die übrigen Autoren geben an, dass kein Interessenkonflikt besteht.

Sponsor der Studie im Sinne des AMG war die Fa. Infectopharm Arzneimittel GmbH, Heppenheim.

Institute

1 Fachkrankenhaus Kloster Grafschaft, Abteilung Pneumologie II,

Schmallenberg

2 St. Elisabeth Krankenhaus, Frankfurt

3 Justus-Liebig Universität Gießen, Med. Klinik II,

${ }^{4}$ LVA-Klinik Donaustauf, Donaustauf

${ }^{5}$ Krankenhaus Bethanien, Solingen

${ }^{6}$ Krankenhaus Bethanien, Moers

Infectopharm Arzneimittel GmbH, Heppenheim

\section{Literatur}

1 Global Initiative for Chronic Obstructive Pulmonary Disease. Global strategy for the diagnosis, management and prevention of chronic obstructive pulmonary disease. Executive Summary 2005: Available at www.goldcopd.com

2 Casaburi R, Mahler DA, Jones PW et al. A long-term evaluation of oncedaily inhaled tiotropium in chronic obstructive pulmonary disease. Eur Respir J 2002; 19: 217 - 24

3 Aaron $S D$, Vandemheen $K L$, Fergusson $D$ et al. Tiotropium in combination with placebo, salmeterol, or fluticasone-salmeterol for treatment of chronic obstructive pulmonary disease. Ann Intern Med 2007; 146: $545-555$

4 Calverley P, Pauwels $R$, Vestbo J et al. Combined salmeterol and fluticasone in the treatment of chronic obstructive pulmonary disease: a randomised controlled trial. Lancet 2003; 361: 449-56 
5 Wedzicha JA, Rabe KF, Martinez FJ et al. Efficacy of roflumilast in chronic obstructive pulmonary disease frequent exacerbator phenotype. Chest 01. 11 2012: 10.1378/chest 12-1489

6 Soler-Cataluna JJ, Martinez-Garcia MA, Roman SP et al. Severe acute exacerbations and mortality in patients with chronic obstructive pulmonary disease. Thorax 2005; 60: 925-931

7 Hill AT. Association between airway bacterial load and markers of airway inflammation in patients with stable chronic bronchitis. Am J Med 2000; 109: $288-295$

8 Patel IS, Seemungal TAR, Wilks $M$ et al. Relationship between bacterial colonisation and the frequency, character and severity of COPD exacerbations. Thorax 2002; 57: 759-764

9 Donaldson GC, Seemungal TAR, Bhowmik A et al. Relationship between exacerbation frequency and lung function decline in chronic obstructive pulmonary disease. Thorax 2002; 57: 847-852

10 Wilkinson TM, Patel IS, Wilks $M$ et al. Airway bacterial load and FEV1 decline in patients with chronic obstructive pulmonary disease. Am J Respir Crit Care Med 2003; 167: 1090-109

11 Murphy TF, Sethi S. Bacterial infection in chronic obstructive pulmonary disease. Am Rev Respir Dis 1992; 146: 1067-1083

12 Geller DE, Pitlick WH, Nardella PA et al. Pharmakokinetics and bioavailability of aerolized tobramycin in cystic fibrosis. Chest 2002; 122: 219-226

13 Ramsey BW, Pepe MS, Quan JM et al. Intermittent administration of inhaled tobramycin in patients with cystic fibrosis. N Engl J Med 1999; 340: $23-30$

14 Ratjen F, Munck A, Kho P et al. Treatment of early Pseudomonas aeruginosa infection in patients with cystic fibrosis: the ELITE Trial. Thorax 2010; 65: $286-291$
15 Ram FS, Rodriguez-Roisin R, Granados-Navarrete A et al. Antibiotics for exacerbations of chronic obstructive pulmonary disease. Cochrane Database Syst Rev 2006; 2: CD004403

16 Drobnic ME, Suñé $P$, Montoro JB et al. Inhaled tobramycin in non-cystic fibrosis patients with bronchiectasis and chronic bronchial infection with Pseudomonas aeruginosa. Ann Pharmacother 2005; 39: 39-44

17 Scheinberg $P$, Shore E. A pilot study of the safety and efficacy of tobramycin solution for inhalation in patients with severe bronchiectasis. Chest 2005; 127: 1420-1426

18 Wilson $R$, Welte T, Polverino $E$ et al. Ciprofloxacin DPI non-cystic fibrosis bronchiectasis: a phase II randomized study. Eur Respir J 20. 12 2012: Epub ahead of print

19 Rademacher J, Welte T. Bronchiektasen - Diagnose und Behandlung. Dtsch Arztebl Int 2011; 108: 809-815

20 Murray MP, Govan JR, Doherty CJ et al. A randomized controlled trial of nebulized gentamicin in non-cystic fibrosis bronchiectasis. Am J Respir Crit Care Med 2011; 183: 491 - 499

21 Albert RK, Connett J, Bailey WC et al. Azithromycin for prevention of exacerbations of COPD. N Engl J Med 2011; 365: 689-98

22 Seemungal TAR, Wilkinson TMA, Hurst JR et al. Long-term erythromycintherapy is associated with decreased chronic obstructive pulmonary disease exacerbations. Am J Respir Crit Care Med 2008; 11: $1139-1147$

23 Patel IS, Vlahos I, Wilkinson TM et al. Bronchiectasis, exacerbation indices, and inflammation in chronic obstructive pulmonary disease. Am J Respir Crit Care Med 2004; 170: 400-407 